\title{
Politique
}

\section{La réforme du système électoral}

\section{Vincent Lemieux et Marie Lavoie}

Numéro 6, automne 1984

La réforme des institutions politiques

URI : https://id.erudit.org/iderudit/040460ar

DOI : https://doi.org/10.7202/040460ar

Aller au sommaire du numéro

Éditeur(s)

Société québécoise de science politique

ISSN

0711-608X (imprimé)

1918-6584 (numérique)

Découvrir la revue

Citer cet article

Lemieux, V. \& Lavoie, M. (1984). La réforme du système électoral. Politique, (6), 33-50. https://doi.org/10.7202/040460ar

Ce document est protégé par la loi sur le droit d'auteur. L’utilisation des services d’Érudit (y compris la reproduction) est assujettie à sa politique d'utilisation que vous pouvez consulter en ligne.

https://apropos.erudit.org/fr/usagers/politique-dutilisation/
Cet article est diffusé et préservé par Érudit.

Érudit est un consortium interuniversitaire sans but lucratif composé de l’Université de Montréal, l'Université Laval et l'Université du Québec à Montréal. Il a pour mission la promotion et la valorisation de la recherche. https://www.erudit.org/fr/ 


\title{
La réforme du système électoral
}

\author{
Vincent Lemieux et Marie Lavoie \\ Université Laval
}

Cet article se fonde sur un jugement de fait et sur un jugement de valeur. Le fait est que les systèmes électoraux, surtout les proportionnels, présentent une grande variété et ont des conséquences diverses qu'il est impossible de réduire à des constantes. Quant à la valeur que nous défendons, c'est celle de la représentation des citoyens. Seuls nous apparaissent acceptables les systèmes électoraux qui accordent aux partis, de façon constante, une proportion de sièges à peu près semblable à la proportion de votes qu'ils ont reçus. Autrement dit, seuls les systèmes électoraux proportionnels ont pour nous une valeur positive de représentation. C'est à l'intérieur de ce sous-ensemble de systèmes électoraux qu'il faut choisir celui qui convient le mieux à une société.

L'article sera divisé en trois parties. Dans un premier temps nous montrerons, en nous inspirant d'un schéma systémique, la grande diversité des sytèmes électoraux. En deuxième lieu nous prétendrons de façon normative que les élections sont faites principalement pour représenter les citoyens, et que la formation et l'exercice du gouvernement doivent être subordonnés à cette exigence première. Enfin, dans une troisième partie, après avoir indiqué les traits principaux de la société politique québécoise, nous proposerons un mode de scrutin proportionnel qui a trop peu retenu l'attention des réformateurs, celui de vote unique transférable. 
Dans la mesure où on peut évaluer sa pratique et ses conséquences, ce mode de scrutin semble apporter une solution de rechange valable au système électoral disproportionnel qui afflige le Canada, le Québec et les autres provinces canadiennes.

\section{La diversité des systèmes électoraux}

Certains détracteurs de la proportionnelle ont tendance, pour des fins de polémique, à réduire de façon excessive la grande diversité des systèmes électoraux. En suivant l'exemple de Duverger ${ }^{1}$, ils s'en tiennent à la distinction entre le scrutin majoritaire à un tour, le scrutin majoritaire à deux tours et la représentation proportionnelle. Pourtant, la diversité de ces systèmes est très grande. On peut construire une typologie plus complète comme celle de Bogdanor $^{2}$, qui distingue systèmes majoritaire et pluralitaire, représentation proportionnelle avec liste et vote unique transférable avec en plus les systèmes mixtes. Et pourtant, même cette classification néglige plusieurs variations inhérentes aux types généraux de systèmes électoraux. Ce n'est pas la somme des modalités d'un système électoral qui lui confere un caractère distinctif mais la combinaison fonctionnelle de ces modalités dans la mise en œuvre du système.

Dans son étude sur les systèmes électoraux et leurs conséquences, $\mathrm{Rae}^{3}$ a distingué trois variables principales: la formule électorale, la façon de voter et l'amplitude des circonscriptions, c'est-à-dire le nombre moyen de sièges à pourvoir par circonscription. On voit que ces variables peuvent être interprétées dans un schéma systémique: la façon de voter est à l'entrée du système et l'amplitude

1. Maurice Duverger, Les partis politiques, Paris, A. Colin, 1951.

2. Vernon Bogdanor, "Conclusion: Electoral Systems and Party Systems" dans Bogdanor et Butler, Democracy and Elections, Cambridge, Cambridge University Press, 1983.

3. Douglas Rae, The Political Consequences of Electoral Laws, New Haven, Yale University Press, 1971. 
des circonscriptions est un aspect important de la sortie, c'est-àdire du nombre relatif de sièges qui seront obtenus par les partis. Quant à la formule électorale, c'est en quelque sorte le processus de conversion des votes en sièges.

La formule électorale peut être pluralitaire au sens où le candidat ayant reçu la majorité des votes est déclaré élu peu importe que cette majorité soit relative ou absolue. Elle peut être majoritaire, c'est-à-dire que le candidat n'est déclaré élu que lorsque la majorité absolue des votes est acquise. La formule électorale peut être également proportionnelle, sans oublier les formules mixtes ou hybrides. Il convient également de prendre en compte diverses modalités de ces formules, soit le seuil de distribution des sièges, la méthode de répartition des restes, la nature des listes lorsqu'elles existent... L'ensemble de ces éléments laisse entrevoir une multitude de formules électorales, toutes aussi disparates les unes que les autres.

Quant à la façon de voter, elle est aussi très diverse. Dans certains systèmes, l'électeur doit exprimer un choix unique. Il est contraint d'indiquer sa préférence pour un candidat ou encore pour un parti à l'exclusion de tous les autres. Rae qualifie ce scrutin de catégorique. Par contre; certaines règles autorisent l'électeur à nuancer son choix, il parle alors de scrutin ordinal. Par exemple, la représentation proportionnelle telle qu'appliquée en Suisse permet à l'électeur de panacher les listes, c'est-à-dire que l'électeur, disposant d'autant de votes qu'il y a de candidats à élire, a la possibilité de les partager entre plusieurs candidats des différents partis. En Irlande, le vote unique transférable autorise l'électeur à ranger les candidats par ordre de préférence. Il en est de même du vote alternatif en vigueur en Australie, à la Chambre des représentants, mais la ressemblance entre ces deux formules se limite à cela; le premier étant un scrutin proportionnel, le second une formule majoritaire. Ces façons de voter ne doivent donc pas être associées au scrutin majoritaire ou à la représentation 
En somme, non seulement les systèmes électoraux sont très divers, mais ils sont, à strictement parler, des systèmes, c'est-àdire des ensembles d'éléments interdépendants, comprenant la façon de voter, la formule de conversion des votes en sièges et l'amplitude des circonscriptions.

\section{La primauté de la représentation}

Il y a différentes façons d'évaluer un système électoral. Dans la conclusion de l'ouvrage collectif publié sous la direction de Bogdanor et Butler ${ }^{4}$, le premier de ces deux auteurs a montré que les conséquences politiques des systèmes électoraux sont nombreuses. Ils peuvent avoir des effets sur le nombre des partis, sur les alliances ou les coalitions entre eux, sur la stabilité gouvernementale, sur la démocratie interne aux partis, sur les stratégies électorales, sur le recrutement politique et sur les relations entre les électeurs et les élus.

Ces différents effets peuvent être ramenés aux trois grandes fonctions des partis, leurs fonctions de représentation, leurs fonctions de gouverne et leurs fonctions de sélection, intermédiaires entre les fonctions de représentations et les fonctions de gouverne. Les relations entre les électeurs et les élus ainsi que le recrutement politique appartiennent aux fonctions de représentation, alors que les alliances et coalitions ainsi que les problèmes de stabilité gouvernementale appartiennent aux fonctions de gouverne. La démocratie interne et les stratégies électorales se rapportent plutôt aux fonctions de sélection, bien qu'elles peuvent être ramenées aux fonctions de représentation ou aux fonctions de gouverne.

La grande opposition entre la représentation et la gouverne soustend la plupart des jugements normatifs qui sont portés sur les systèmes électoraux. Malheureusement, on s'en tient le plus

4. Vernon Bodganor et David Butler (eds), op. cit. 
souvent aux aspects formels de la représentation et de la gouverne. Nous avons montré, dans un autre article ${ }^{5}$ qu'en plus des aspects strictement quantitatifs de la représentation, il fallait aussi tenir compte de sa qualité, et qu'au-delà des notions vides de stabilité ou d'efficacité gouvernementale, il fallait aussi considérer la «responsabilité» d'un gouvernement, au sens de sa capacité à répondre aux attentes ou aux exigences du public. Cela complique l'évaluation des conséquences d'un système électoral. Elle se complique encore plus si on prend en compte les fonctions de sélection des partis, c'est-à-dire leur capacité à orienter les activités gouvernementales ou les activités de représentation dans le public.

Nous adoptons et défendons ici une position normative qui simplifie quelque peu l'évaluation des systèmes électoraux. Pour nous, en effet, les valeurs de la représentation ont la primauté sur celles de la gouverne. Un système électoral a pour fin de permettre la représentation équitable des électeurs et non pas de former un gouvernement. Nous nous proposons d'ailleurs de montrer qu'en l'absence d'une représentation équitable la stabilité et l'efficacité gouvernementale ne sont que des illusions, magnifiées par ceux qui ont intérêt à une stabilité gouvernementale sans contenu ou à la prétendue efficacité gouvernementale, parce qu'ils ont pris le parti des gouvernants contre celui des gouvernés.

La démocratie représentative n'est pas un mécanisme pour assurer la suprématie des gouvernants sur les gouvernés, au prix de la représentation disproportionnelle du parti le plus nombreux. C'est un mécanisme pour informer l'appareil gouvernemental de la variété des partis et du poids respectif des électeurs qui les appuient. Le gouvernement doit composer avec cette variété et non pas en faire l'économie par la sous-représentation excessive des regroupements d'électeurs autres que le plus nombreux, ou

5. Marie Lavoie et Vincent Lemieux, "L'évaluation des modes de scrutin", Revue parlementaire canadienne, vol. $6, \mathrm{n}^{\circ} 4,1983-1984$. 
les deux plus nombreux. Cela déplaît aux intellectuels d'élite qui préferent les modes gouvernementales aux courants sociaux. C'est ainsi que le rédacteur de l'Actualité écrit (mai 1984, page 10):

«La proportionnelle favorise la floraison des partis de nuisance. Elle tendrait à faire de l'Assemblée une pétaudière de toutes les nuances de l'arc-en-ciel, du Crédit social aux Rhinocéros, en passant par les Raéliens, les jovialistes, les verts et tous (sic) les "particules» d'une seule idée."

De ce point de vue, le Parti québécois était un parti de nuisance, à la fin des années 60 .

Il est difficile de considérer qu'un système électoral est fonctionnel, en démocratie représentative, quand la moitié - et parfois plus - des électeurs sont représentés au Parlement par un député pour lequel ils n'ont pas voté. C'est pourtant la situation habituelle avec le scrutin majoritaire à un tour.

Signalons en passant que ce système électoral se justifie mieux dans un régime présidentiel comme celui des États-Unis que dans un régime parlementaire à l'anglaise. Le président ne peut être élu qu'au scrutin majoritaire, de même que les gouverneurs à la tête des États et les sénateurs qui ne sont élus qu'un à la fois. Quant aux membres de la Chambre des représentants, ils sont élus à tous les deux ans. Cette vitesse de roulement atténue les inconvénients du système majoritaire. Plus généralement, l'absence de discipline de vote et le pouvoir des groupes de pression amènent les élus à représenter plus que les électeurs qui ont voté pour eux.

Qu'il soit tempéré ou non par d'autres processus de représentation, le système électoral majoritaire est fondé sur une conception irréelle de la démocratie. Dans les petites collectivités dont rêvait Rousseau, les électeurs qui constatent, en prenant connaissance des résultats, qu'ils sont minoritaires, reconnaissent leur erreur et se rallient à la majorité, porteuse de la volonté générale. Maịs dans la société pluraliste, où ne règne plus l'amitié sociale chère à Rousseau, l'opération majoritaire est un leurre. 
Elle laisse une grande proportion des votants, et parfois même la majorité d'entre eux, sans représentant dans le territoire découpé pour fin d'élection. Elle a dressé abusivement, à la Chambre des Communes, les libéraux du Québec contre les conservateurs de l'Ouest, sans que des conservateurs du Québec ou des libéraux de l'Ouest puissent, par leur présence et leur action, atténuer ces conflits. Elle fait croire de façon tout aussi abusive aux gouvernements en place, quand ils comptent leurs députés, qu'ils sont la majorité alors qu'ils n'ont pas pour eux la majorité des votants.

Ces vices seraient compensés et même effacés, aux yeux des partisans du système majoritaire, par les vertus de la stabilité gouvernementale, gage d'efficacité gouvernementale. Bogdanor a fait une critique dévastatrice du gouvernement des partis, en Grande-Bretagne, en montrant comment la distorsion de la représentation, due au scrutin majoritaire, expliquait une bonne partie des errements dans les politiques gouvernementales ${ }^{6}$. Plusieurs éléments de sa critique s'appliquent également à la situation canadienne et québécoise.

Avec le système majoritaire, non seulement le parti de gouvernement est porté à surestimer les appuis qu'il a dans la population, mais il est aussi porté à surévaluer, dans la fabrication des politiques, les milieux d'où viennent ses appuis les plus denses. Par exemple, un parti qui est appuyé à $55 \%$ dans les grands centres urbains et à $45 \%$ seulement dans les régions périphériques aura la très grande majorité de ses députés dans les grands centres et quelquesuns seulement dans la périphérie, même s'il y a autant d'électeurs dans ces milieux qu'au centre. Ce parti sera tout naturellement porté à gouverner en fonction des intérêts du centre et pourra même dresser, par son action, la périphérie contre le centre. Bien sûr, ces excès ont plus de chances de se produire dans une société

6. Vernon Bodganor, The People and the Party System, Cambridge, Cambridge University Press, 1981. 
qui est divisée à la base et quand l'action d'un parti de gouvernement est programmatique. Mais, ce qui importe de noter, c'est que ces excès sont en quelque sorte inscrits dans la logique des systèmes électoraux majoritaires, alors qu'ils ne le sont pas dans la logique des systèmes proportionnels.

Il demeure que représenter est une chose et que gouverner en est une autre, comme l'ont bien vu ceux qui ont réfléchi sur ces phénomènes ${ }^{7}$. De notre point de vue, qui est normatif, on ne doit pas tricher avec la représentation, mais il faut aussi assurer, autant que possible, que la gouverne qui s'élève sur une représentation équitable soit non pas tant efficace qu'adéquate. L'efficacité est une notion vide qui, lorsqu'on la remplit, renvoie aux objectifs du gouvernement et à la fabrication de politiques conformes à ces objectifs. La notion d'adéquation est plus exigeante. Elle renvoie à d'autres critères que ceux que se donne un gouvernement. La gouverne est adéquate quand elle répond aux attentes des publics, c'est-à-dire à des critères extérieurs, dont on accepte qu'ils puissent changer, malgré soi. C'est une autre façon de dire que la gouverne doit être «répondante» ou démocratique, au sens de la belle définition donnée par Schattschneider ${ }^{8}$, quand il disait de la démocratie qu'elle était un système pour des gens qui n'étaient pas tout à fait sûrs d'avoir raison.

Dans cette perspective, le problème est donc de se donner un système électoral qui, tout en assurant une représentation équitable, permette - avec bien d'autres institutions et pratiques - que la gouverne soir adéquate.

Le système électoral doit être ajusté à un système politique et à une société donnée, qui sont des ensembles bien plus déterminants d'un système partisan et d'un mode de gouverne que le Press, 1967.

7. Hanna Pitkin, The Concept of Representation, Berkely, University of California

8. E. E. Schattschneider, Tuo Hundred Million Americans in Search of a Government, New York, Holt, Rinehard and Winston, 1969. 
système électoral. Il est ridicule d'écrire, comme on le fait trop souvent, que la proportionnelle (sans qu'on précise laquelle) créerait au Canada ou au Québec des situations semblables à celle de l'Italie ou à celle de la France de la IV République. Ces affirmations polémiques manifestent une science sociale déficiente, pour ne pas dire nulle. Il est tout aussi contestable d'attribuer à la seule proportionnelle les réussites politiques relatives (elles le sont toujours...) de la République fédérale d'Allemagne, des pays scandinaves ou de la Suisse.

Ce n'est donc quaprès avoir établi les principaux traits pertinents d'une société politique qu'on peut dessiner les modalités de proportionnelle qui favoriseraient la gouverne adéquate de cette société.

\section{Une proportionnelle pour une gouverne adéquate}

C'est un exercice simplificateur que de réduire une société politique à quelques traits principaux. Mais cela vaut mieux que de la considérer comme un milieu indifférencié où toutes les conséquences positives ou négatives attribuées aux systèmes proportionnels peuvent se produire, selon les besoins de la polémique.

Du Québec et, dans une certaine mesure, du Canada, on peut dire qu'il présente, d'un point de vue comparatif, au moins les quatre caractéristiques politiques suivantes:

$1^{\circ}$ À l'exception des clivages ethniques et linguistiques, et peutêtre des clivages régionaux, les divisions de nature sociétale ne sont pas particulièrement grandes. Il y a bien, à tous les 30 ou 35 ans, émergence de phénomènes de génération (avec l'Union Nationale puis le Parti québécois), mais les divisions idéologiques ne sont pas très profondes.

$2^{\circ} \quad$ Le régime politique est de nature parlementaire, c'est-à-dire que la direction du gouvernement appartient au parti ou à 
une éventuelle coalition de partis qui peut compter sur une majorité des appuis au Parlement.

$3^{\circ} \quad$ Les grands partis sont généralement centrés autour de leurs dirigeants, comme un peu partout à travers le monde, mais leur degré d'organisation est relativement faible, ce qui en fait des milieux moins rigides et qui peuvent davantage être ouverts au public que la plupart des grands partis européens. $4^{\circ}$ Le rôle d'intermédiaire du député entre les électeurs et l'appareil gouvernemental demeure valorisé, même s'il ne procure plus autant d'avantages qu'autrefois. Ce n'est pas là un trait universel. En République fédérale d'Allemagne et dans les pays scandinaves, ce rôle d'intermédiaire n'existe à peu près pas. Par contre, les députés participent plus activement à la législation.

Si ces traits sont exacts, beaucoup d'arguments utilisés contre l'introduction d'un système proportionnel au Québec ou encore au palier fédéral ne sont pas pertinents.

Il est bien peu probable que la proportionnelle entraîne la multiplication des partis. Il faudrait pour cela que la société soit plus divisée qu'elle l'est actuellement. Des petits partis apparaîtraient peut-être mais leurs chances de faire élire plus de quelques députés seraient restreintes surtout si, comme c'est probable, des circonscriptions ont une faible amplitude.

On prétend souvent que la proportionnelle favorise la centration du pouvoir entre les mains des états-majors partisans, par la capacité qu'ont ceux-ci d'établir les listes de candidats et l'ordre des candidats sur ces listes. Cela est vrai là où les dirigeants ont effectivement ce pouvoir et où les électeurs ne peuvent voter que pour des listes. Il n'est pas nécessaire qu'il en soit ainsi. Si les électeurs peuvent exprimer des votes préférentiels et "panachés» pour les candidats et que ceux-ci sont choisis en des congrès d'investiture démocratiques, largement ouverts aux membres des partis, un système proportionnel peut être moins centralisateur 
qu'un système majoritaire, comme l'enseigne d'ailleurs le cas de la Suisse, parmi d'autres. Il y a eu beaucoup de progrès qui ont été faits dans la démocratisation des partis du Québec depuis 25 ans, malgré des accidents de parcours, si bien qu'à l'heure actuelle ils ne sont pas particulièrement centrés, si on les compare à d'autres. Nous sommes prêts à leur accorder une confiance relative pour l'avenir, qu'il y ait système proportionnel ou non.

Enfin, la soi-disant sauvegarde, par le scrutin majoritaire, du lien personnel entre les électeurs et leur élu comporte plusieurs illusions. Il faut d'abord rappeler que beaucoup de députés, en système majoritaire, ont été choisis par moins de la moitié des votànts et qu'il est relativement rare que les électeurs des partis perdants aient recours au député. Plus généralement, des sondages nous ont appris que c'est environ $15 \%$ des électeurs, en moyenne, qui disent entrer en contact avec leur député. Comme nous venons de le signaler, ces contacts ne sont plus aussi avantageux qu'avant (dans les grandes années du patronage...) pour les deux partis. À une époque où les députés sont apportés puis emportés par des vagues de fond qui les dépassent, il est illusoire de penser que les contacts avec les électeurs augmentent les chances de réélection. Et surtout, il nous semble encore plus illusoire de vouloir sauver la vocation d'intermédiaire du député et, en même temps, de réformer le parlement pour donner plus de pouvoir législatif aux parlementaires. Les expériences étrangères enseignent que le député ne peut être à la fois un intermédiaire efficace et un législateur actif. C'est non seulement une question de disponibilité, mais aussi une question de talent et de compétence. De façon générale, les bons intermédiaires ne font pas les bons législateurs et les bons législateurs ne font pas les bons intermédiaires.

Reste le problème du régime parlementaire et de la difficulté qu'il y a à assurer une gouverne adéquate à partir d'une représentation équitable. Une solution consisterait à changer le régime parlementaire pour un régime présidentiel ou encore pour un régime 
à la suisse, où un conseil exécutif de sept personnes de différents partis, nommées par l'Assemblée, gouverne le pays.

Mais supposons, de façon réaliste, que le régime parlementaire actuel ne sera pas modifié. Il faut alors choisir, nous semble-t-il, un système proportionnel qui donne une petite prime au parti qui obtient le plus de votes, de façon à augmenter la probabilité qu'il forme seul le gouvernement.

Dans la perspective d'une gouverne adéquate, les gouvernements de coalition ont beaucoup de qualités, à condition qu'ils ne soient pas constants. Les coalitions occasionnelles rendent les gouvernements plus attentifs aux attentes'des gouvernés parce qu'il y a alors au moins deux lectures (et parfois plus) de ces attentes. Mais, quand les coalitions sont constantes, les négociations entre les responsables des partis risquent de finir par être décrochées des attentes des publics. C'est pourquoi l'alternance entre gouvernement d'un seul parti et gouvernement de coalition semble être une meilleure solution que la solitude constante ou la coalition constante. L'expérience des régimes parlementaires européens où existent des systèmes proportionnels va dans ce sens. Signalons qu'en République fédérale d'Allemagne, tout au moins, les sondages d'opinion ont montré constamment que les électeurs préferent les gouvernements de coalition aux gouvernements d'un seul parti ${ }^{9}$.

La représentation proportionnelle régionale modérée (dite RPRM) et la version quelque peu modifiée, dite de représentation territoriale, qui a été proposée par la Commission de la représentation électorale, en mars 1984, fournissent selon nous les garanties nécessaires à une représentation équitable et à une gouverne adéquate.

Il y a toutefois un autre système proportionnel qui donne ces garanties et qui serait bien ajusté à la société politique québécoise. C'est le système du vote unique transférable (VUT). On peut

9. Peter Pulzer, "Germany» dans V. Bodganor et D. Butler, op. cit., 102. 
d'ailleurs s'étonner qu'aucun intervenant ne l'ait proposé à la Commission de la représentation électorale, lors des audiences de l'automne 1983. Nous voudrions, pour finir, souligner certaines qualités trop peu connues de ce système électoral. Il est utilisé en République d'Irlande, mais aussi dans l'île de Malte, dans l'État australien de la Tasmanie et pour l'élection des sénateurs en Australie.

Des réformateurs l'écartent en disant qu'il est trop compliqué alors qu'au contraire les Irlandais le trouvent facile à comprendre. Ils ont d'ailleurs refusé deux fois, par voie de référendum, en 1959 et en 1968, de le remplacer par le scrutin majoritaire, même si les gouvernements du temps leur recommandaient de le faire.

Le vote unique transférable s'exerce dans des circonscriptions comportant quelques sièges (de 3 à 5 en Irlande). Les partis présentent un ou plusieurs candidats, selon leurs espoirs de réussite, et les électeurs votent pour ces candidats en exprimant un ordre de préférence entre eux. On compte d'abord les premiers choix et un ou des candidats sont élus s'ils atteignent le quota requis. Ce quota est établi selon la formule suivante:

$$
\frac{\text { nombre de suffrages }}{\text { nombre de sièges }+1}+1
$$

Par exemple, si 10000 suffrages ont été exprimés et qu'il y a trois sièges à pourvoir, le quota sera de 2501 . Généralement, quelques candidats seulement, ou même aucun, sont élus de la sorte. Si aucun des candidats n'est élu dans un premier temps, on élimine (comme on le fait à nos congrès au leadership) le candidat qui a obtenu le moins de premiers choix et on attribue les deuxièmes choix des électeurs qui l'ont appuyé aux candidats vers lesquels ces choix se sont portés. Si l'un des candidats obtient alors le quota, il est élu. Et l'on procède de la sorte jusqu'à ce que tous les sièges soient pourvus, ou bien à ceux qui ont atteint 
le quota ou bien, à défaut de cela, à celui ou ceux qui ont obtenu le plus de votes.

Si un ou des candidats atteignent le quota avec les premières préférences des électeurs, les secondes préférences des électeurs qui ont voté pour ces candidats sont d'abord redistribuées, avant que l'on procède à l'élimination des candidats de dernier rang.

Donnons un exemple simple, mettant en présence les candidats $A$ et $B$ d'un premier parti, et les candidats $X, Y$ et $Z$ d'un second parti. Les 10000 votes de premier choix des électeurs se sont répartis de la façon suivante:

$$
\begin{aligned}
& \mathrm{A}=3000 \\
& \mathrm{~B}=1900 \\
& \mathrm{X}=2300 \\
& \mathrm{Y}=1500 \\
& \mathrm{Z}=1300
\end{aligned}
$$

Le candidat $A$ a atteint le quota, qui est de 2501 , comme nous l'avons vu plus haut. Il est donc déclaré élu. On tient compte alors des secondes préférences des électeurs qui ont voté pour ce candidat et on les répartit sur une base de 249 votes, soit l'excédent de A par rapport au quota. Supposons qu'aucun autre candidat n'atteint le quota suite à cette redistribution. Z, le candidat ayant obtenu le moins de premières préférences, est alors éliminé et les deuxièmes préférences des électeurs qui l'ont appuyé sont distribuées aux trois autres candidats restants. $\mathrm{X}$, supposons-le, atteint ainsi le quota (il ne lui manquait que 151 votes pour ce faire élu. Il ne reste plus que $\mathrm{B}$ et $\mathrm{Y}$. Le troisième siège est accordé à celui des deux qui a obtenu le plus de votes suite aux opérations préalables, dont la redistribution de l'excédent de X. Ce sera sans doute $B$ qui obtiendra ainsi le troisième et dernier siège.

Notons que si le second parti n'avait présenté que deux candidats plutôt que trois, il aurait pu obtenir deux sièges sur 
trois. Cela montre l'importance stratégique du choix des candidats par les partis ou encore de la dissidence à l'intérieur d'un parti.

Les spécialistes ${ }^{10}$, qui ont étudié les conséquences de ce système électoral en République d'Irlande estiment généralement qu'il ne permet qu'une emprise restreinte des organisations centrales des partis sur les candidats, puisqu'il n'y a pas de listes au sens strict et que les candidats d'un même parti ne sont pas ordonnés entre eux. Le vote unique transférable n'a pas conduit à une multiplication des partis car il n'y a que trois partis importants en République d'Irlande malgré des clivages qui sont certainement aussi importants qu'au Québec. Les électeurs envoient plutôt des "messages" aux partis existants, d'une élection à l'autre, en déplaçant leurs premiers choix à l'intérieur d'un même parti. C'est pour cela un système électoral plus transparent que la plupart des autres. Il est aussi très proportionnel, même s'il donne généralement une petite prime en sièges au parti qui obtient le plus de votes.

Enfin, ce système n'a pas empêché que se maintiennent des relations très personnelles entre les élus et les électeurs. La République d'Irlande a un système politique où le député est d'abord un intermédiaire, un "ombudsman" et même un patron pour plusieurs de ses électeurs. Toutefois, c'est aux dépens de son rôle de législateur.

Il pourrait exercer ce rôle davantage s'il n'était pas aussi accaparé par son rôle d'intermédiaire, qui le rend d'ailleurs plus ou moins prisonnier des instances supérieures de son parti de qui dépend le succès de ses médiations.

Toutefois, comme le note Bogdanor ${ }^{11}$, il n'est pas dit que, dans une société où le rôle du député serait défini autrement, le

10. Basil Chubb, «The Electoral System" dans H. R. Penniman (ed.) Ireland at the Polls, Washington, American Enterprise Institute for Public Policy, 1978; V. Bodganor, op. cit., 1981; Paul Mckee "The Republic of Ireland" dans V. Bodganor et D. Butler op. cit., 1983.

11. V. Bodganor, op. cit., 1981, 248. 
système du vote unique transférable favoriserait quand même le rôle d'intermédiaire. L'accent mis sur les rôles du député semble plutôt indépendant du système électoral. La Grande-Bretagne, les pays scandinaves et la République fédérale d'Allemagne ont des systèmes électoraux très différents les uns des autres et pourtant, dans tous les cas, le député est davantage un législateur qu'un intermédiaire.

\section{Conclusion}

De notre point de vue, la réforme du système électoral doit être fondée sur la primauté de la représentation. C'est seulement quand les partis en lice reçoivent de façon constante une proportion de sièges à peu près égale à leur proportion de votes, et que le très grand nombre des électeurs sont représentés par des députés du parti pour lequel ils ont voté, que la démocratie représentative peut fonctionner de façon convenable. Cela est encore plus vrai à une époque où l'on exigera des gouvernements qu'ils soient responsables plutôt que «forts», et adéquats plutôt que seulement efficaces.

On peut puiser dans la grande diversité des systèmes électoraux et en particulier des systèmes proportionnels pour trouver une solution de rechange au système majoritaire qui soit ajustée à notre société et qui rende improbable le caractère constant des gouvernements de coalition: Il ne faudrait toutefois pas croire que les gouvernements de coalition n'ont que des défauts. Ils ont, au contraire, beaucoup de qualités quand ils alternent avec les gouvernements d'un seul parti. De toute façon, quand les électeurs n'optent pas de façon majoritaire ou quasi-majoritaire pour un des partis, mieux vaut un gouvernement de coalition que le gouvernement d'un parti, qui est entrâ̂né, de façon illusoire, à agir comme s'il était plus populaire que ses adversaires réunis. 
L'étude des conséquences des systèmes électoraux est complexe et pourtant elle donne lieu à beaucoup de simplifications et de jugements abusifs. Sans cacher les valeurs qui nous inspirent, nous avons voulu, dans cet article, contester quelques-unes de ces simplifications et remettre en question certains de ces jugements, pour ensuite proposer de façon plus constructive un système électoral, celui du vote unique transférable, qui n'a pas suffisamment retenu l'attention des intervenants dans les débats récents sur la réforme du système électoral. 